\title{
Early Experience Shapes Amygdala Sensitivity to Race: An International Adoption Design
}

\author{
Eva H. Telzer, ${ }^{1,2}$ Jessica Flannery, ${ }^{2}$ Mor Shapiro, ${ }^{2}$ Kathryn L. Humphreys, ${ }^{2}$ Bonnie Goff, ${ }^{2}$ Laurel Gabard-Durman, ${ }^{2}$ \\ Dylan D. Gee, ${ }^{2}$ and Nim Tottenham ${ }^{2}$ \\ ${ }^{1}$ Department of Psychology, University of Illinois at Urbana-Champaign, Urbana, Illinois 61801, and 2Department of Psychology, University of California, \\ Los Angeles, Los Angeles, California 90095
}

In the current study, we investigated how complete infant deprivation to out-group race impacts behavioral and neural sensitivity to race. Although monkey models have successfully achieved complete face deprivation in early life, this is typically impossible in human studies. We overcame this barrier by examining youths with exclusively homogenous racial experience in early postnatal development. These were youths raised in orphanage care in either East Asia or Eastern Europe as infants and later adopted by American families. The use of international adoption bolsters confidence of infant exposure to race (e.g., to solely Asian faces or European faces). Participants completed an emotional matching task during functional MRI. Our findings show that deprivation to other-race faces in infancy disrupts recognition of emotion and results in heightened amygdala response to out-group faces. Greater early deprivation (i.e., later age of adoption) is associated with greater biases to race. These data demonstrate how early social deprivation to race shapes amygdala function later in life and provides support that early postnatal development may represent a sensitive period for race perception.

\section{Introduction}

Humans show an advantage for perceiving and understanding faces and emotions of individuals within their own cultural group (Elfenbein and Ambady, 2002; Pascalis et al., 2005; Kelly et al., 2007; Scott and Monesson, 2009; Vogel et al., 2012). One view holds that, through a process of perceptual narrowing, environmental inputs tune the brain toward the predominant category at a cost to non-experienced stimuli (Kelly et al., 2007; Sugita, 2008; Dahl et al., 2012). Early-life experiences may be particularly influential in tuning neurobiological mechanisms involved in face recognition (Furl et al., 2002; Sangrigoli et al., 2005). It is perhaps through this tuning process that neural regions, such as the amygdala, manifest differential responses to racial categories observed in adulthood (Hart et al., 2000). This neural tuning process may give rise to other-race perceptual biases, often referred to as the other-race effect (ORE; Vogel et al., 2012).

The ORE is thought to be the end result of a disproportionately high degree of exposure to one's own cultural group compared with other groups (Walker and Tanaka, 2003). Despite the theorized role of experience in tuning the face-processing system, experience with race is especially hard to quantify in racially/ ethnically diverse places, such as the United States, perhaps explaining why other-race contact has accounted for $<3 \%$ of the

\footnotetext{
Received March 20, 2013; revised July 6, 2013; accepted July 11, 2013.

Author contributions: E.H.T. and N.T. designed research; E.H.T., J.F., M.S., K.H., B.G., L.G.-D., D.G.G., and N.T. performed research; E.H.T. and N.T. analyzed data; E.H.T. and N.T. wrote the paper.

This work was supported by National Institute of Mental Health Grant R01MH091864 (N.T.).

Correspondence should be addressed to Eva H. Telzer, Department of Psychology, 603 E. Daniel, M/C 716, University of Illinois at Urbana-Champaign, Urbana, IL 61820. E-mail: ehtelzer@illinois.edu.

DOI:10.1523/JNEUROSCI.1272-13.2013

Copyright $\odot 2013$ the authors $\quad 0270-6474 / 13 / 3313484-05 \$ 15.00 / 0$
}

total variance in the ORE (Meissner and Bringham, 2001). The amygdala is highly sensitive to environmental influence, particularly early in life when it undergoes rapid development (Payne et al., 2010; Gee et al., 2013). Therefore, it is possible that the modulation of amygdala response by race observed in adolescence and adulthood as well as the ORE (Hart et al., 2000; Lieberman et al., 2005; Telzer et al., 2013) reflects the history of neural tuning by early-life social experiences.

Although monkey models have successfully achieved complete face deprivation paradigms in early life (Sugita, 2008), this is typically impossible in most human studies, and little is known about how early experience shapes face recognition and contributes to the specialization of neural structures underlying face processing. In the current study, we overcome this barrier by examining youths with exclusively homogenous racial experience in early postnatal development. These were youths raised in orphanage care in either East Asia or Eastern Europe as infants. Orphanage rearing represents a socially deprived rearing environment in general, but importantly for the design of this study, prevents exposure to other-race faces. The youths in this study were adopted by American families, and, because age of adoption is documented, this design provides a natural index of the amount of early experience with a specific racial group and the amount of deprivation to other face types. This quasiexperimental design via international adoption bolsters confidence of infant exposure to race (e.g., to solely Asian faces or solely European faces). We measured internationally adopted youths' emotion recognition of in- and out-group faces using functional magnetic resonance imaging (fMRI) to test the hypothesis that complete infant deprivation to out-group faces would be followed by a perceptual tuning bias away from the out-group. 


\section{Materials and Methods}

Participants. Forty-nine youths participated, including 17 youths adopted from Asian orphanages (mean age of 11.2 years, age range of 6.516.4 years; mean age adopted of 25.8 months, range of 6-90 months; 14 females), 19 youths adopted from European orphanages (mean age of 9.9 years, age range of 6.3-15.6 years; mean age adopted of 36.45 months, range of 8-120 months; 9 females), and 13 European-American nonadopted youths (mean age of 10.8 years, age range of $7-16$ years; 9 females) who lived with their biologically related parents. Asian- and European-adopted children did not differ in their mean age or age of adoption. All children were adopted by European-American families in the United States. European-American, non-adopted youth were from the Los Angeles metropolitan area.

Emotional matching task. Participants completed a task during fMRI, in which they selected emotional faces that matched a target face (Telzer et al., 2013). Participants were presented with a trio of faces and were instructed to make a button response to indicate which of the two faces at the bottom was expressing the same emotion, or felt the same, as the target face on top. The target faces were displaying happy or angry facial expressions, and all were taken from the NimStim Set of Facial Expressions (Tottenham et al., 2009). Participants completed two counterbalanced functional runs blocked by race (Asian or European). Each run consisted of 12 faces, which were each presented for $5 \mathrm{~s}$. Run order was counterbalanced across participants.

fMRI data acquisition. Participants were scanned on a Siemens Trio 3.0 Tesla MRI scanner. For each participant, an initial 2D spin echo image (TR, $4000 \mathrm{~ms}$; TE, $40 \mathrm{~ms}$; matrix size, $256 \times 256$; $4 \mathrm{~mm}$ thick; $0 \mathrm{~mm}$ gap) in the oblique plane was acquired to enable prescription of slices obtained in the structural and functional scans. A whole-brain, highresolution, $\mathrm{T}^{*}$-weighted anatomical scan (MP-RAGE; $192 \times 192$ inplane resolution; $256 \mathrm{~mm}$ FOV; $192 \times 1 \mathrm{~mm}$ sagittal slices) was acquired for each subject for registration and localization of functional data into Talairach space. The emotional matching task was presented on a computer screen through MR-compatible goggles. The task was completed during two functional scans. Ninety-nine $\mathrm{T} 2^{\star}$-weighted echo planar images were collected (TR, 2000; TE, $30 \mathrm{~ms}$; flip angle, $90^{\circ}$; matrix size, $64 \times$ 64; 34 slices; $4 \mathrm{~mm}$ voxel; skip $0 \mathrm{~mm}$ ) at an oblique angle of $\sim 30^{\circ}$.

$f M R I$ data analyses. Functional imaging data were preprocessed and analyzed with the Analysis of Functional NeuroImages (AFNI) software package (Cox, 1996). All data were free of movement $>2.5 \mathrm{~mm}$ in any direction. Preprocessing for each participant's images included slice time correction to adjust for temporal differences in slice acquisition within each volume, spatial realignment to correct for head motion, registration to the first volume of each run, spatial smoothing using anisotropic $6 \mathrm{~mm}$ Gaussian kernel, full-width at half-maximum to increase the signal-tonoise ratio, and transformation into the standard coordinate space of Talairach and Tournoux (Talairach and Tournoux, 1988) with parameters obtained from the transformation of each subject's high-resolution anatomical scan. Talairached transformed images had a resampled resolution of $3 \mathrm{~mm}^{3}$. Time series were normalized to percentage signal change to allow comparisons across runs and individuals by dividing signal intensity at each time point by the mean intensity for that voxel and multiplying the result by 100 .

The functional runs were concatenated before creating each participant's individual-level model by convolving the stimulus timing files with canonical hemodynamic response function. Six motion parameters were included as separate regressors. General linear modeling was performed to fit the percentage signal change time courses to each regressor. Linear and quadratic trends were modeled in each voxel time course to control for correlated drift. The individual-level regression coefficients were submitted to random-effects, group-level analyses. We conducted a linear mixed effects analyses within AFNI to examine group differences in neural activation to the contrast of Asian versus European faces. Age, IQ, and age of adoption were included as covariates. Correction for multiple comparisons was applied at the cluster level after Monte Carlo simulations conducted with $3 \mathrm{dClustSim}$ within AFNI. This method controls for type I errors, offering a reasonable correction for multiple tests during group-level analyses in regions of interest. Clusterwise false-positive rates of $p<0.05$, small-volume corrected with a minimum cluster size of nine voxels, were used for the bilateral amygdala (Phan et al., 2006). Non-a priori regions outside of the amygdala were corrected for multiple comparisons within the whole brain at $p<0.01$, with a minimum cluster size of 56 voxels. All analyses control for age and IQ (Wechsler Abbreviated Scale of Intelligence; Wechsler, 1999).

\section{Results}

\section{Behavioral results}

To examine group differences in behavioral performance to matching the emotional expression of in- and out-group faces, we ran repeated-measures ANOVA to examine response time and accuracy to identifying Asian and European faces, with group (Asian-adopted, European-adopted) as a between-subjects factor and age and IQ entered as covariates.

\section{Response time}

Mean response times (MRTs) for identifying the emotion of each face type were relatively fast, such that participants responded in $<1.5$ of $5 \mathrm{~s}$ possible across both face types (Asian faces, MRT \pm $\mathrm{SD}, 1.4 \pm 0.6 \mathrm{~s}$; European faces, MRT $\pm \mathrm{SD}, 1.3 \pm 0.5 \mathrm{~s})$. A repeated-measures ANOVA examining group differences in response times to identifying the emotion of Asian and European faces, controlling for age and IQ, revealed no significant effects for MRT, such that participants' response times did not differ for identifying the emotion of Asian and European faces, and there was not a significant interaction of group $\times$ face type, suggesting that there are not differential response times to identifying emotions of in- and out-group faces.

\section{Error rate}

Error rate was calculated by taking the number of errors for each face type and dividing by the total number of faces in that condition. Overall error rates were relatively low (mean $\pm \mathrm{SD}$; Asian faces, $5.3 \pm 9.2 \%$; European faces, $4.3 \pm 7.4 \%$ ). A repeatedmeasures ANOVA examining accuracy to identifying the emotion of Asian versus European faces, with group as a between-subjects factor, controlling for age and IQ, revealed no significant main effect, suggesting that, overall, participants show similar accuracy rates for identifying the emotion of Asian and European faces. However, this was qualified by a significant interaction for group, such that youths were less accurate at identifying the emotion of out-group relative to in-group faces $\left(F_{(1,32)}=7.4, p<0.01\right)$. For visual purposes, we calculated differential error rates to Asian and European faces by subtracting the error rate for European faces from the error rate for Asian faces. Positive scores indicate greater errors when identifying the emotion of Asian relative to European faces, scores $\sim 0$ indicate no differential bias, and negative scores indicate greater errors to identifying the emotion of European relative to Asian faces. As shown in Figure $1 a$, Asian- and European-adopted children showed differential accuracy, such that they were less accurate at identifying emotional expressions in the other race compared with their own race. As a comparison, European-American children's behavioral response is depicted on the top of Figure 1a. European-American children do not show a differential response to identifying the emotional expressions of Asian and European faces.

\section{Age of adoption}

To examine whether youths show a differential response to identifying emotional expressions to in- and out-group faces as a function of the amount of early deprivation to race, we examined age of adoption whereby Asian-adopted children's age of adoption was reverse scored, such that the continuous variable ranged from negative scores, representing prolonged early deprivation of 
a
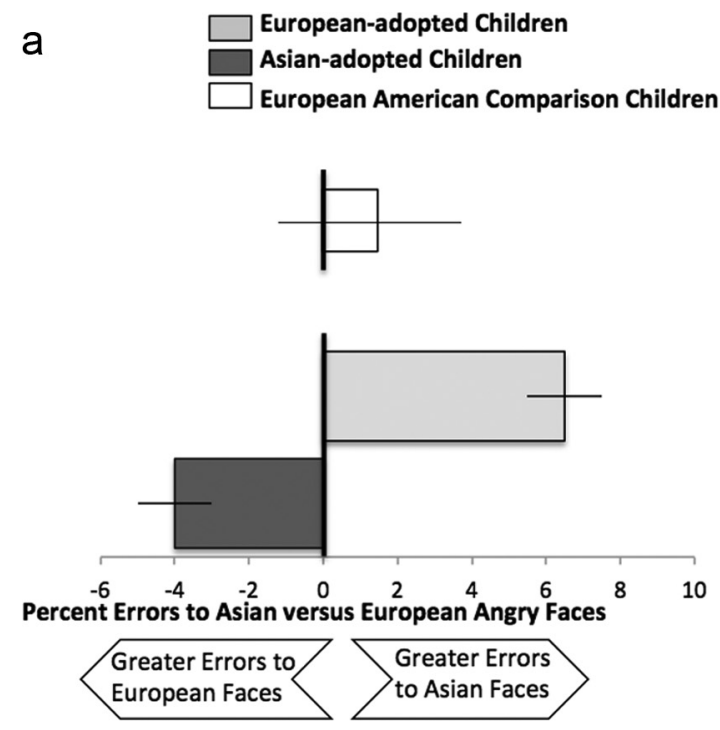

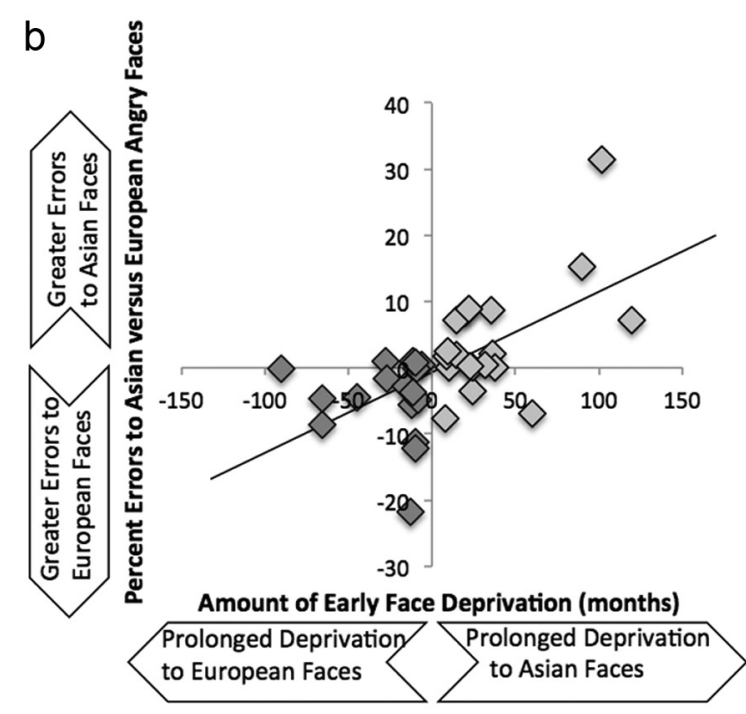

Figure 1. a, Behavioral performance during the emotional expression matching game. Asian-adopted and European-adopted children show differential accuracy to in- and out-group faces, such that they are each more accurate at identifying emotional expressions of in-group faces than out-group faces. For descriptive purposes only, European-American children are depicted at the top of the figure in white. European-American children do not demonstrate a differential response to identifying emotional expressions of in- and out-group faces. $\boldsymbol{b}$, Age of adoption is associated with accuracy to identifying emotional expressions of in- versus out-group faces. Greater early race deprivation is associated with greater differential performance.

European faces, to positive scores, representing prolonged early deprivation of Asian faces. Scores $\sim 0$ indicate little deprivation to out-group faces. A multiple-regression analysis was run in which the differential error rate to identifying the emotion of Asian and European faces was predicted by age of adoption, controlling for age and IQ. Results indicate that greater early deprivation was associated with greater differential performance to identifying emotional expressions of in-group relative to out-group faces $(\beta=0.57, p<0.001$; Fig. $1 b)$.

\section{fMRI results}

To examine group differences in neural responses to race, a linear mixed-effects model was run in AFNI with group (Asianadopted, European-adopted) predicting neural response to Asian versus European faces with age, IQ, and age of adoption as covariates. A significant group effect was found in the bilateral amygdala to Asian versus European faces [right amygdala: $F_{(4,32)}=13.47, p<0.05$ corrected, $(x, y, z)=(13,-3,-13,39)$, $k=39$ contiguous voxels; left amygdala: $F_{(4,32)}=10.99, p<0.05$ corrected, $(x, y, z)=(-12,-3,-14), k=194$ contiguous voxels], such that European-adopted children showed greater bilateral amygdala response when identifying emotional expressions to Asian faces, whereas Asian-adopted children showed greater amygdala activation when identifying emotional expressions to European faces (Fig. 2a). As a comparison, European-American children's amygdala response is depicted on the top of Figure $2 a$. European-American children do not show a differential response in the amygdala when identifying emotional expressions to Asian versus European faces. No other brain regions showed a significant group effect to Asian versus European faces, even at a lowered threshold of $p<0.05$ uncorrected.

Age of adoption

To examine whether youths show a differential amygdala response when identifying the emotions of in- and out-group faces as a function of the amount of early deprivation, we extracted the percentage signal change from the amygdala cluster that showed a significant group effect. Regression analyses in SPSS show that greater early deprivation to race is associated with differential amygdala response to in-group relative to out-group faces $(\beta=$ 0.54, $p<0.001$; Fig. $2 b$ ).

\section{Brain-behavior relationships}

In our final analysis, we ran a regression analysis in SPSS to examine whether amygdala activation is related to behavioral performance during the emotional matching task. We used the cluster of amygdala that showed a significant group effect to Asian versus European faces and ran a regression analysis predicting differential accuracy to identifying the emotion of Asian and European faces, controlling for age and IQ. Participants who showed heightened differential amygdala response demonstrated greater differential accuracy $(\beta=0.49, p<0.005)$. As shown in Figure 3 , participants with greater relative amygdala activation to Asian faces than European faces showed greater relative errors when matching the emotional expression of Asian relative to European faces and vice versa.

\section{Discussion}

The current study investigated how early postnatal deprivation to race affects behavioral and amygdala sensitivity to race. Using a unique international adoption design, we could, in a controlled manner, measure how experience with face types influences behavioral and neural processing of emotions of in- and out-group faces. Our findings show that early experience with racial categories tunes face emotion processing toward the more familiar face category, also known as the ORE. We found that complete deprivation to out-group faces early in development was followed by lower recognition accuracy and higher amygdala activation to out-group faces relative to in-group faces. This bias toward ingroup faces is associated with the amount of early race deprivation; earlier age of adoption was associated with an attenuated ORE, including higher relative accuracy at identifying expressions of out-group faces as well as reduced amygdala sensitivity. Importantly, differential amygdala response to race was associated with differential behavioral performance to identifying the emotions of in- and out-group faces, suggesting that neural biases to race may manifest behaviorally, highlighting the impactful role that differential amygdala sensitivity plays. 

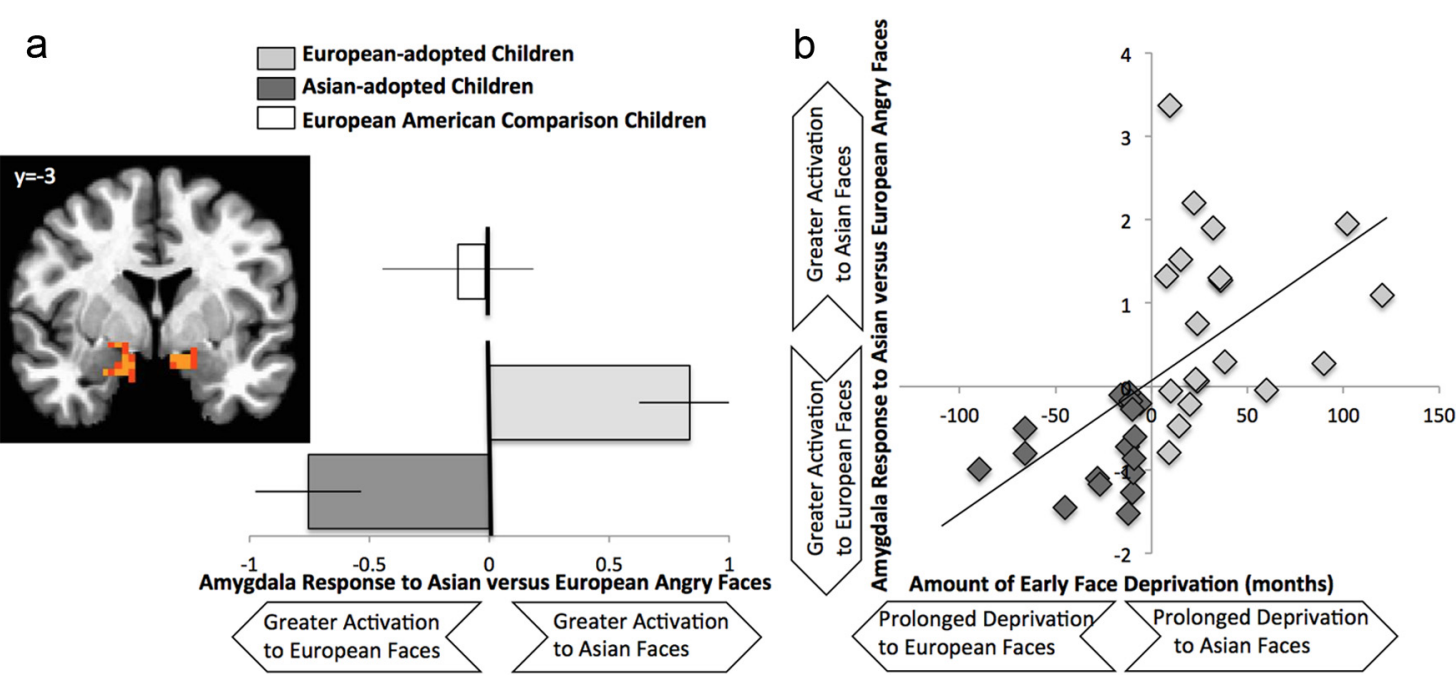

Figure 2. $\quad \boldsymbol{a}$, Group differences in the bilateral amygdala to Asian versus European faces during the emotional expression matching game. Asian-adopted and European-adopted youths show differential amygdala response when identifying emotional expressions of in- and out-group faces, such that each group shows heightened amygdala response to out-group faces compared with in-group faces. For descriptive purposes only, European-American children are depicted at the top of the figure. European-American children do not demonstrate differential amygdala response when identifying emotional expressions of Asian versus European faces. Note that right $=$ left. $\boldsymbol{b}$, Age of adoption is associated with greater amygdala differentiation when identifying the emotional expression of in-versus out-group emotional faces. Greater early race deprivation is associated with greater amygdala response to the out-group compared with in-group. Note that age and IQ are controlled for in all analyses and figures.

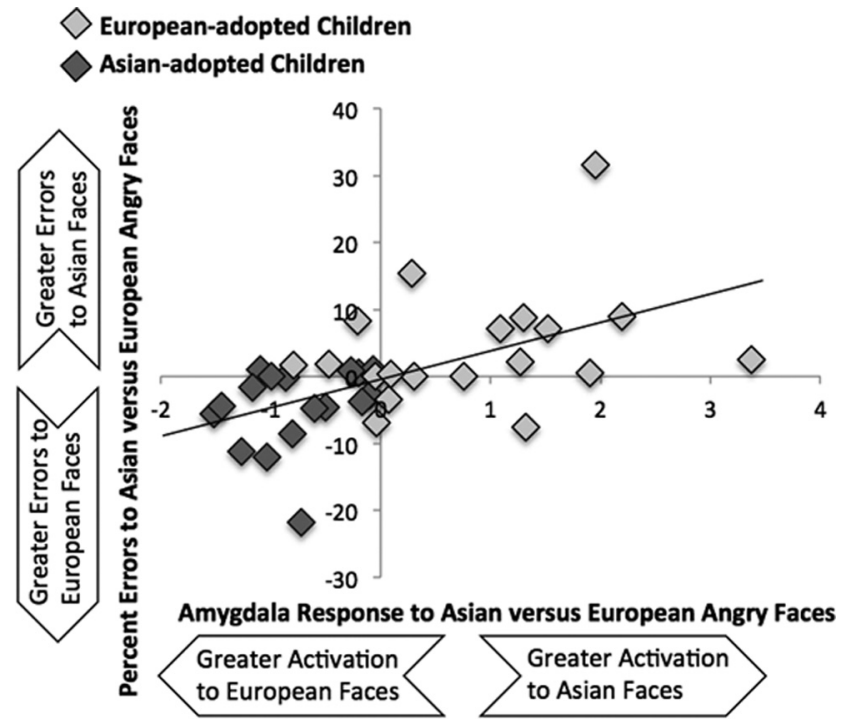

Figure 3. Correlating amygdala activation to behavioral performance. Greater differential amygdala activation to Asian and European faces is related to greater differential accuracy to identifying the emotional expressions of Asian and European faces.

The amygdala is an early developing brain region (Payne et al., 2010) that is sensitive to emotionally and motivationally salient stimuli in one's environment (Whalen et al., 2001; Cunningham and Brosch, 2012), as well as the detection of novel and unusual stimuli (Blackford et al., 2010). Therefore, the heightened amygdala response observed in the current study may suggest that out-group faces are both relatively novel and particularly salient. These findings are consistent with adult work showing stronger amygdala signal to other-race faces relative to own-race faces (Hart et al., 2000; Krill and Platek, 2009), suggesting that other-race faces are particularly salient, perhaps as a result of novelty or unfamiliarity with the out-group. Saliency itself has been shown to increase amygdala responding; saliency may be high during the initial learning phase of in-group membership as has been shown with minimal-group paradigms in adulthood (Van Bavel et al., 2008). With increased experience, saliency for in-group versus out-group members may switch as the outgroup members remain less familiar and the in-group members become more familiar. This perspective is consistent with the perceptual narrowing hypothesis and suggests that early experiences are particularly important for shaping the tuning properties of the system. Thus, the amygdala becomes culturally biased toward or away from racial categories as a function of early exposure to own-race relative to other-race faces. Early deprivation to out-group race renders out-group faces more novel and salient, resulting in poorer emotion recognition and enhanced amygdala responsivity.

Our findings are consistent with previous work showing that infants show differential processing of in- and out-group faces. For instance, Vogel et al. (2012) found that, by 9 months, infants do not differentiate among faces of the other race, show a decline in the ability to match emotions in other-race faces, and demonstrate differential neural processing to in-group relative to outgroup faces. Similar results have been found in monkeys deprived of faces, such that, during the first year of life, monkeys exposed to one species of faces (e.g., human or monkey) at the cost of the other species show a marked decline in the ability to recognize the non-exposed faces (Sugita, 2008). Together, our findings suggest that the ability to perceive and recognize facial expressions at a level that allows for generalization across racial types develops during the first year of life, and deprivation to other-races (or species) results in a marked decline in the processing of outgroup faces.

Early postnatal development may be a sensitive period whereby lack of early exposure to out-group faces limits the encoding of these faces later in development (Sangrigoli et al., 2005). Indeed, early postnatal development is an important temporal window during which experience warps the face representational system; once in place, the system limits the encoding of new faces and is less sensitive to new inputs (Furl et al., 2002). Thus, face perception may reflect an experience-expectant process, such that exposure to faces during early postnatal develop- 
ment leads to perceptual (i.e., recognition bias) and neural (i.e., amygdala desensitization) specialization at the cost of nonexperienced stimuli (Nelson, 2001). Our findings provide strong evidence for the perceptual narrowing hypothesis of race perception, such that selective experience with own-race versus otherrace faces early in life tunes the system toward the predominant category, leading to a decline in the ability to recognize emotional expressions in other-race faces (Sangrigoli et al., 2005; Kelly et al., 2007; Sugita, 2008; Dahl et al., 2012).

Importantly, youths who had greater early deprivation (i.e., later age of adoption) showed the strongest differential bias to own- and other-race faces, suggesting that the amount of deprivation and the timing at which children are exposed to new faces can affect the tuning of the face perceptual system. Such differential tuning of the face perceptual system leads to greater emotional arousal to other-race faces. Although youths in this study were exposed to other races by childhood, it is possible that the encoding of new faces is already limited by childhood, resulting in a deficit in recognizing emotional expressions in out-group faces. Indeed, European adults who receive extensive interracial contact with Asian individuals (e.g., European individuals living in Singapore) do not show an improved ability to recognize the faces from the other race ( $\mathrm{Ng}$ and Lindsay, 1994). Together with our findings, this suggests that the age at which experience with other race faces begins is a crucial factor in face perception and emotional recognition of other-race faces (Sangrigoli and De Schonen, 2004).

Of note, our sample of adopted youths is individuals who spent time in institutionalized care as infants. Although this type of rearing provides a racially homogenous environment for youths, institutionalized care is a non-normative developmental experience associated with impairments in emotional processing and heightened amygdala reactivity (Tottenham and Sheridan, 2009). In addition, our control group consisted of EuropeanAmerican children who had not experienced social deprivation. Although our findings suggest that these non-deprived youths do not show differential behavioral or neural biases to in- and outgroup faces, a better control would have consisted of EuropeanAmerican children who had been adopted from within the United States. This control group would have better controlled for the general effects of early adverse care giving. Nonetheless, our findings demonstrate differential responses to in- and outgroup faces among youths who experienced early race deprivation. We have no reason to speculate that adverse rearing environments would result in differential processing of race. However, future work that identifies individuals with documentable race deprivation in the absence of social deprivation would confirm this assumption.

In conclusion, our findings highlight the role of experience in the development of the ORE. Complete deprivation to other-race faces in infancy disrupts recognition of emotion and results in heightened amygdala response to out-group faces. These data provide support that early postnatal development may represent a sensitive period for emotion recognition of out-group faces.

\section{References}

Blackford JU, Buckholtz JW, Avery SN, Zald DH (2010) A unique role for the human amygdala in novelty detection. Neuroimage 50:1188-1193. CrossRef Medline

Cox RW (1996) AFNI: software for analysis and visualization of functional magnetic resonance neuroimages. Comput Biomed Res 29:162-173. Medline

Cunningham WA, Brosch T (2012) Motivational salience. Amygdala tuning from traits, needs, values, and goals. Curr Dir Psychol Sci 21:54-59. CrossRef

Dahl CD, Rasch MJ, Tomonaga M, Adachi I (2013) Developmental processes in face perception. Sci Rep 3:1044. CrossRef Medline

Elfenbein HA, Ambady N (2002) On the universality and cultural specificity of emotion recognition: a meta-analysis. Psychol Bull 128: 203-235. CrossRef Medline

Furl N, Phillips PJ, Toole AJ (2002) Face recognition algorithms and the other-race effect: computational mechanisms for a developmental contact hypothesis. Cognit Sci 26:797-815. CrossRef

Gee DG, Humphreys KL, Flannery J, Goff B, Telzer EH, Shapiro M, Hare TA, Bookheimer SY, Tottenham N (2013) A developmental shift from positive to negative connectivity in human amygdala-prefrontal circuitry. J Neurosci 33:4584-4593. CrossRef Medline

Hart AJ, Whalen PJ, Shin LM, McInerney SC, Fischer H, Rauch SL (2000) Differential response in the human amygdala to racial outgroup vs ingroup face stimuli. Neuroreport 11:2351-2355. CrossRef Medline

Kelly DJ, Quinn PC, Slater AM, Lee K, Ge L, Pascalis O (2007) The otherrace effect develops during infancy: evidence of perceptual narrowing. Psychol Sci 18:1084-1089. CrossRef Medline

Krill A, Platek SM (2009) In-group and out-group membership mediates anterior cingulate activation to social exclusion. Front Evol Neurosci 1:1. CrossRef Medline

Lieberman MD, Hariri A, Jarcho JM, Eisenberger NI, Bookheimer SY (2005) An fMRI investigation of race-related amygdala activity in AfricanAmerican and Caucasian-American individuals. Nat Neurosci 8:720722. CrossRef Medline

Meissner CA, Brigham JC (2001) Thirty years of investigating the own-race bias in memory for faces: a meta-analytic review. Psychol Publ Pol Law 7:3-35. CrossRef

Nelson CA (2001) The development and neural bases of face recognition. Inf Child Dev 10:3-18. CrossRef

$\mathrm{Ng}$ W, Lindsay RC (1994) Cross-race facial recognition: failure of the contact hypothesis. J Cross Cult Psychol 25:217-232. CrossRef

Pascalis O, Scott LS, Kelly DJ, Shannon RW, Nicholson E, Coleman M, Nelson CA (2005) Plasticity of face processing in infancy. Proc Natl Acad Sci U S A 102:5297-5300. CrossRef Medline

Payne C, Machado CJ, Bliwise NG, Bachevalier J (2010) Maturation of the hippocampal formation and amygdala in Macaca mulatta: a volumetric magnetic resonance imaging study. Hippocampus 20:922-935. CrossRef Medline

Phan KL, Fitzgerald DA, Nathan PJ, Tancer ME (2006) Association between amygdala hyperactivity to harsh faces and severity of social anxiety symptoms in generalized social phobia. Biol Psychiatry 59:424-429. CrossRef Medline

Sangrigoli S, De Schonen S (2004) Recognition of own-race and otherrace faces by three-month-old infants. J Child Psychol Psychiatry 45: 1219-1227. CrossRef Medline

Sangrigoli S, Pallier C, Argenti AM, Ventureyra VA, de Schonen S (2005) Reversibility of the other-race effect in race recognition during childhood. Psychol Sci 16:440-444. CrossRef Medline

Scott LS, Monesson A (2009) The origin of biases in face perception. Psychol Sci 20:676-680. CrossRef Medline

Sugita Y (2008) Face perception in monkeys reared with no exposure to faces. Proc Natl Acad Sci U S A 105:394-398. CrossRef Medline

Talairach J, Tournoux P (1988) Co-planar stereotactic atlas of the human brain. New York: Thieme.

Telzer EH, Humphreys KL, Shapiro M, Tottenham N (2013) Amygdala sensitivity to race is not present in childhood but emerges in adolescence. J Cogn Neurosci 25:234-244. CrossRef Medline

Tottenham N, Sheridan MA (2009) A review of adversity, the amygdala and the hippocampus: a consideration of developmental timing. Front Hum Neurosci 3:68. CrossRef Medline

Tottenham N, Tanaka JW, Leon AC, McCarry T, Nurse M, Hare TA, Marcus DJ, Westerlund A, Casey BJ, Nelson C (2009) The NimStim set of facial expressions: judgments from untrained research participants. Psychiatry Res 168:242-249. CrossRef Medline

Van Bavel JJ, Packer DJ, Cunningham WA (2008) The neural substrates of in-group bias: a functional magnetic resonance imaging investigation. Psychol Sci 19:1131-1139. CrossRef Medline

Vogel M, Monesson A, Scott LS (2012) Building biases in infancy: the influ- 
ence of race on face and voice emotion matching. Dev Sci 15:359-372. CrossRef Medline

Walker PM, Tanaka JW (2003) An encoding advantage for own-race versus other-race faces. Perception 32:1117-1125. CrossRef Medline

Wechsler D (1999) Wechsler Abbreviated Scale of Intelligence (WASI). San Antonio, TX: Harcourt Assessment.
Whalen PJ, Shin LM, McInerney SC, Fischer H, Wright CI, Rauch SL (2001) A functional MRI study of human amygdala responses to facial expressions of fear versus anger. Emotion 1:70-83. CrossRef Medline 